subsequent urinary tract infection (UTI) (patient self-report, GP records or antibiotic prescription).

Results Management approved the study and received strong support from both staff and participants showing high levels of altruism. This was due to high levels of engagement, communication and a clear plan as to what was being done and why, how this was to be achieved and how success would be measured. Additionally, staff felt the sheathed cystoscope handled well.

Overall, 1091 endosheathed flexible cystoscopies were analysed. It was a very well tolerated procedure with $33.2 \%$ procedures causing no discomfort, $48.2 \%$ mild and only $3.1 \%$ severe discomfort.

The UTI rate was $13.3 \%$ and impressively there were no reports of urosepsis.

Conclusions This safe, well tolerated procedure provides both cost and time savings to the healthcare provider. This study paves the way for developments in endoscopic imaging techniques and can be used as a leading example for other hospitals to implement the new techniques. This is also translatable to other units performing diagnostic telescopic evaluations in other parts of the body (e.g. bronchoscopy).

\section{IMPROVING PHYSICAL HEALTHCARE FOR INPATIENTS ON A PSYCHIATRIC UNIT}

${ }^{1}$ Emma Watura*, ${ }^{2}$ David Denton. 'University Hospitals Sussex NHS Foundation Trust, UK. Lewisham and Greenwich NHS Foundation Trust, UK; ${ }^{2}$ Sussex Partnership Foundation Trust, UK. Oxleas NHS Foundation Trust, UK

\subsection{6/leader-2021-FMLM.34}

Background Good quality physical healthcare provision for patients with a serious mental illness can be challenging. Healthcare staff may feel they lack the knowledge and support to manage their patients' physical healthcare needs. Patients, therefore, may receive reduced quality of care for their medical conditions whilst an inpatient on a psychiatric unit.

Aims To identify areas for improvement of physical healthcare provision for inpatients on a unit for patients with psychiatric disorders.

Method A closed-loop audit was conducted on an inpatient psychiatry unit in West Sussex, UK. Retrospective data was manually collected from patient information systems. All new admissions between 14th January - 15th May 2021 were included for the first audit cycle (21 patients). After educational intervention, a second audit cycle was completed including all new admissions between 23rd May - 23rd July 2021 (18 patients).

Results The first audit cycle showed that $86 \%$ of patients had a physical examination and $76 \%$ had a venous thromboembolism (VTE) assessment within 24 hours of admission. It took an average of 13 days for the admission blood tests to be taken and, on average, 19 days for completion of admission electrocardiogram (ECG). Five patients did not have an ECG throughout admission. A reason for this was documented for four of these patients.

Following educational intervention, the percentage of patients having a physical examination and VTE assessment within 24 hours of admission increased by $8 \%$ and $24 \%$ respectively. The average number of days for admission blood tests and ECG completion decreased by 12 days and 13 days, respectively.
Conclusion The improvements made during this audit process may hasten the detection and treatment of medical pathology. Overall, this improves patient care and aids avoidance of the complications of delayed diagnosis or treatment.

*Corresponding author and presenter.

\section{INTEGRATED MEDICAL LEADERSHIP EXPERIENCE FOR JUNIOR DOCTORS}

Nelomi Anandagoda*, Jennifer Tringham, Olushola Alonge. Frimley Health NHS Foundation Trust, Portsmouth Road, Camberley, GU16 7UJ

\subsection{6/leader-2021-FMLM.35}

The surge in admissions during the COVID-19 pandemic necessitated implementation of an incident rota for $>130$ junior doctors (JD) including new/re-deployed doctors. Conscious of the risk of feeling underprepared, isolated or lost in a big re-shuffle, JD were allocated to 5 teams each with a varied skill mix. Five registrars and ten SHOs, were identified by senior clinical leaders as showing leadership potential and appointed as 'Team Leaders' (TL).

TL promoted psychological well-being, provided peer-support for their team and managed rotas to ensure safe staffing across 30 wards. The chief registrar met weekly with TL to support leadership challenges and maintain an oversight of the expanded multi-speciality workforce. Supporting new leaders to be accessible and creating a support network allowed a large-scale change in working patterns, whilst maintaining a localised, team-based feel.

Prior to nomination, $44.4 \%$ of TL had no leadership experience. Therefore, a self-assessment survey covering the five domains of the medical leadership framework (personal qualities, team working, managing services, improving services and setting direction) was carried out to assess the impact of this experience.

The majority of respondents felt that they had achieved leadership skills covering all five domains. 100\% felt that their TL experience made them more likely to seek future leadership roles with $66.5 \%$ considering applying for a specific leadership training programme e.g. RCP Chief Registrar and Darzi Fellow programmes.

As a result of the positive impact of this unique leadership opportunity an allocated weekly session has been incorporated into IMT3 job plans, with the aim of continuing to support and empower junior doctors to pursue senior leadership roles. Equipping trainees with the requisite technical and leadership skills to directly influence change throughout their careers and develop into tomorrow's clinical leaders will undoubtedly improve patient outcomes.

\section{\begin{tabular}{|l|l}
\hline 6 & INTRAOSSEOUS CONTRAST INJECTION SERVICE IN
\end{tabular} ABERDEEN ROYAL INFIRMARY}

Yajur Narang*, Senthil Ragupathy. Aberdeen Royal Infirmary, UK

\subsection{6/leader-2021-FMLM.36}

Background Establishing rapid intravenous (IV) access during initial resuscitation in trauma patients is vital for optimum quality CT scan examination. However, this can be challenging and at times futile. Reasons like severe haemorrhage, obesity, 
burns, dehydration, prior IV chemotherapy, and IV drug use make peripheral veins difficult to identify and cannulate.

Critical time can be lost while attempting to obtain vascular access, potentially increasing patient morbidity and mortality. On the other hand, central venous access is time-consuming and poses complication risks like infections and pneumothorax.

In these instances, Intra-osseous (IO) contrast injections are beneficial to obtain good quality trauma scans for patients from the Emergency Department (ED).

Methods Multi-professional stakeholders (Radiologists, ED consultants, and Radiographers) met to identify the need for IO service in Aberdeen Royal Infirmary. This was followed by discussions at the CT user's group meeting. A need for developing a local protocol for IO contrast service was identified for patients with no IV access. A draft protocol was created and then modified to meet local needs.

Interventions Once the protocol was finalised, IO contrast service was introduced for trauma patients without IV access. The local protocol gives clear information about placement and checking of IO needle, local anaesthetic administration and the technique for contrast administration.

Results Since implementing the protocol, 3 successful trauma scans have been performed using IO access. The quality of images has been good with no recorded complications.

Conclusions IO contrast provides an effective and safe alternative to IV access. Good quality images can be obtained using established protocols.

Presenting author Dr Yajur Narang*

\section{IS THERE A NEED TO CHANGE THE DELIVERY OF LEADERSHIP TRAINING TO OUR FUTURE DOCTORS?}

Jabin Chowdhury, Victor Kimani, Haroon Ali Shah. UK

10.1136/leader-2021-FMLM.37

The General Medical Council (GMC) has advocated for wider leadership and management responsibilities for doctors and the Birmingham Medical Leadership Society (BMLS) aims to address ways in which students and doctors can be supported through this. Collecting data on this topic is pivotal to improving the way in which medical leadership is taught to prepare students to take on leadership roles.

The survey conducted addressed the importance of leadership training. After analysing 122 responses, the consensus is that medical leadership training is important with $61.5 \%$ of people rating it 5 on the Likert scale. Participants were given the opportunity to rate how they thought medical education would best be learned. $77.9 \%$ agreed an NHS paired learning scheme where students are paired up with a practising healthcare professional, would be a suitable method. Teaching from and shadowing of NHS managers proved popular, as did leadership workshops. The lack of representation of foundation doctors and those further on in their training has created a need for more data from doctors of different grades, to see if their views on medical leadership significantly differ.

Should leadership teaching be introduced earlier on in medical school through the methods

BMLS anticipates that earlier and better medical leadership education will make more confident doctors with capacity to take on leadership roles. Shifting the leaders of healthcare services towards those with clinical background prioritises patients at the centre of care.

Using student views to tailor medical education feels productive to producing the next generation of doctors who are better equipped with skills required to take on leadership roles. BMLS hopes medical schools will listen to these views and take action and that medical leadership training is emphasised in Good Medical Practice. This serves our healthcare service which in turn makes for better quality and safety of care.

\section{LEADERSHIP IN THE MANAGEMENT OF PATIENTS WITH MULTIPLE CONDITIONS: AN IMPACT EVALUATION OF BMJ BEST PRACTICE COMORBIDITIES}

Kieran Walsh. BMJ Knowledge Centre, BMJ, UK

\subsection{6/leader-2021-FMLM.38}

BMJ Best Practice Comorbidities has been created by the BMJ Knowledge Centre. This work was carried out by BMJ staff and was done in collaboration with healthcare professionals in training in the UK. One in three adults admitted to hospital in the UK have five or more conditions. People with comorbidities have poorer functional status, quality of life, and health outcomes, and are higher users of ambulatory and inpatient care than those without comorbidities. This poses a problem for patients, healthcare professionals, health leaders, and health systems. In light of this, BMJ Best Practice launched a new tool - BMJ Best Practice Comorbidities. This tool enables healthcare professionals to access detailed guidance on how to manage patients with multiple conditions and thus provide holistic care. In a pilot evaluation, we made this tool available to a group of healthcare professionals in training. We then asked them to fill in a simple questionnaire outlining what difference, if any, the tool made to their practice. We measured the effect of any improvement as a result of using the tool by means of a short questionnaire. We asked the healthcare professionals to describe the circumstances in which they had used the tool, whether the tool made any difference to their practice, and what impact this had on patient care. The evaluation showed that BMJ Best Practice Comorbidities is effective at helping healthcare professionals to improve the care that they provide to patients with multiple conditions (the resource helped users to improve practice in $70 \%$ of cases). When it doesn't change practice, it can still have an effect by reassuring users that their practice is correct (this occurred in $15 \%$ of cases). This evaluation showed impact in improving the practice of healthcare professionals in delivering high-quality and safe care for patients with comorbidities.

\section{LOCAL TRAINING PROGRAMME FOR ANAESTHETISTS AND FUTURE LEADERS}

Gregory Fraser, Emma James. Imperial College Healthcare Trust, Royal National Orthopaedic Hospital NHS Trust

10.1136/leader-2021-FMLM.39

Primary issues - centralised training programme by HEE for anaesthetics, limited number of training posts compared to 